\title{
Arsine gas poisoning: the importance of exchange transfusions in severe cases
}

\author{
C S HESDORFFER, ${ }^{1}$ F J MILNE, ${ }^{2}$ J TERBLANCHE, ${ }^{3}$ AND A M MEYERS ${ }^{1}$
}

\begin{abstract}
From the Division of Nephrology, ${ }^{1}$ Department of Medicine, Johannesburg Hospital and University of the Witwatersrand Medical School, Division of Nephrology, ${ }^{2}$ Coronation Hospital, and Metal Refinery, ${ }^{3}$ Johannesburg, South Africa
\end{abstract}

Arsine (arsineuretted hydrogen, AsH3) was first identified in 1775. It is a colourless, non-irritant gas that smells like garlic. After the initial demonstration of its toxicity in 1815,454 cases of poisoning had been documented by $1974 .{ }^{1}$ To date, a further 16 cases have been described, ${ }^{2-5}$ including one with permanent renal damage. ${ }^{6}$ Many cases probably go unrecognised as the attending medical staff may not be aware of the possibility of arsine gas poisoning. This may lead to delay in the diagnosis and management with increased mortality and morbidity.

The prognosis of patients exposed to arsine gas depends on the effect on renal function. Previously anuria was a common cause of death but, with dialysis, patients should not die from renal failure. A problem arises with the adequate removal of arsine and its associated toxic complexes. In this respect dialysis alone is insufficient, and only the removal of the arsine haemoglobin haptoglobin complex by exchange transfusion will stop the toxic process.

Several causes for the renal failure have been postulated by Muehrcke and Pirani. ${ }^{7}$ Firstly, arsine gas itself has a direct toxic effect on renal tissue. Secondly, as a result of haemolysis, haemoglobinuric casts may precipitate in the tubules resulting in tubular damage. Finally, the oxygen carrying capacity of blood is reduced by haemolysis and thus hypoxic damage to renal tissue may occur. The case we describe is of a worker at a metal refinery near Johannesburg; he was one of five cases of arsine poisoning seen at Johannesburg Hospital over the past six years.

\section{Methods}

Haemoglobin was measured by Coulter counter. Reticulocyte counts were done by hand and measurement of haptoglobin and haemopexin was performed by electroimmunodiffusion. Liver function tests and the urea and creatinine were measured by autoanalyser. Lactate dehydrogenase was measured automatically by means of the Multistat machine. Blood and urine arsenic concentrations were meaAccepted 29 July 1985 sured at the refinery's laboratory with prior preparation of the sample using the wet oxidation technique. Final results were obtained by means of a hydride atomic absorption reading. The patient was initially dialysed with a UDM machine using a Gambro $120 \mathrm{M}$ dialyser to remove fluid. Once he began diuresing a Gambro 120L dialyser was used.

\section{Case report}

A 36 year old, previously completely well, white man was working on a metal reduction process with hydrochloric acid on 1 March 1983. Six to eight hours after completing the procedure he passed frankly red urine, shown later to contain haemoglobin. The patient felt nauseous and vomited profusely. His output of urine was appreciably decreased and he was referred to Johannesburg Hospital on 3 March. On admission he complained of pronounced abdominal pain and generalised muscle pain and tenderness. Slight pallor and jaundice were noted. His vital signs were normal. No cyanosis was present, and there was no evidence of respiratory distress. A small quantity of "dirty coloured" urine was obtained by means of a catheter, which on dipstix testing was positive for blood but on microscopy no red cells were seen. Later, during the recovery phase, red cells and granular casts were seen. The table shows his blood results in detail. The patient became totaly anuric and only passed good volumes of urine on day 16. Haemodialysis was begun on day 2 . No exchange transfusions were carried out initially because haemolysis, on the basis of the reticulocyte count, did not appear to be severe, and there was a two day delay in obtaining the haptoglobin and haemopexin concentrations from the laboratory. The blood arsenic concentrations fell from $0.6 \mathrm{mg} / 1$ to $0.35 \mathrm{mg} / \mathrm{l}$ over the first eight days, during which he was dialysed four times. His symptoms of abdominal, renal and muscle pain did not improve, however, over this period. Exchange transfusions were then performed on days 10 and 12 with a more rapid fall in the arsenic concentrations, dramatic improvement in his symptoms, and in- 
Laboratory results (normal values in parentheses)

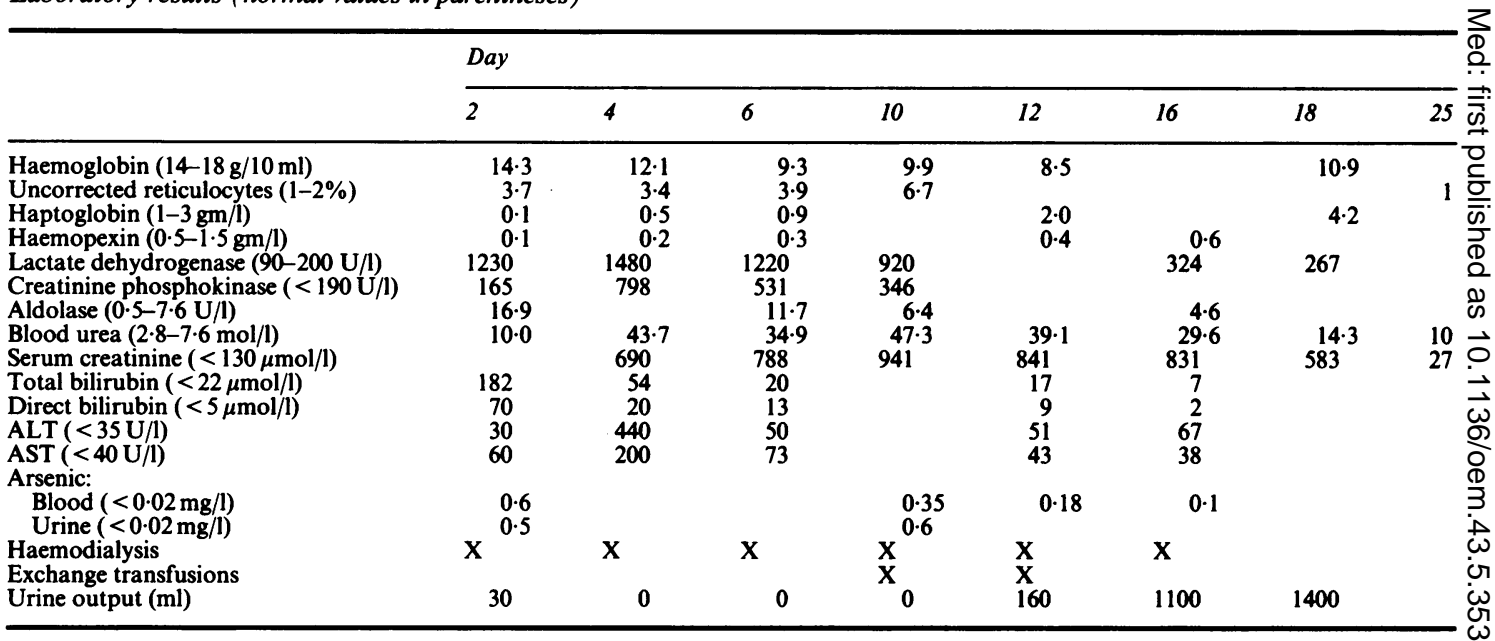

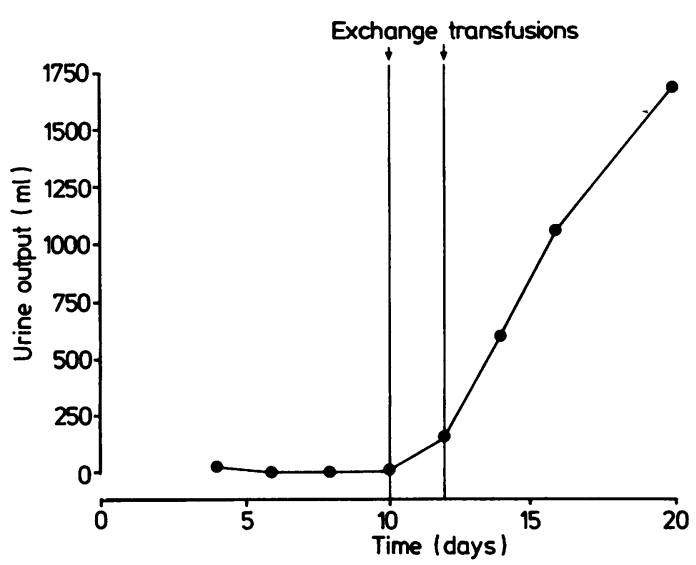

Fig 1 Increasing urine output after exchange transfusions.

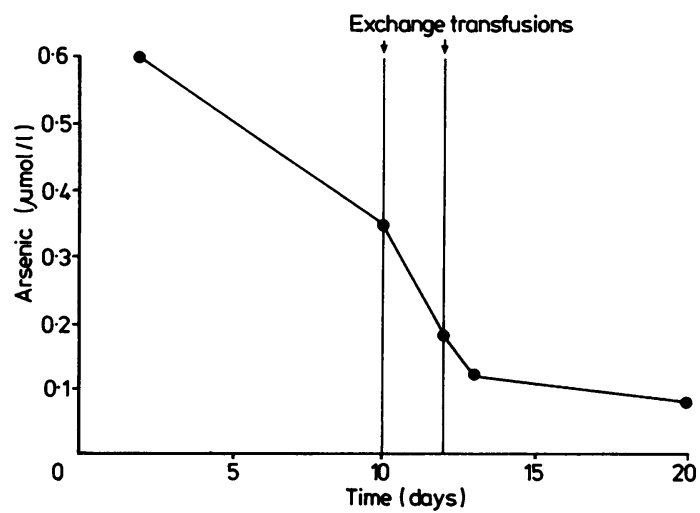

Fig 2 Increased rate of drop of serum arsenic levels after exchange transfusions. creasing output of urine (figs 1 and 2). Although renal $\stackrel{\circ}{\supset}$ recovery may be expected on day 10 after the onset of $\rightarrow$ acute tubular necrosis, the serum creatinine concen- $z$ tration should only have fallen later. As both the blood arsenic and serum creatinine concentrations fell $\vec{\varphi}$ simultaneously we believe this resulted from the ex- $\infty$ change transfusions with the removal of the toxic arsine complexes.

\section{Discussion}

The major effect of arsine is related to its ability to $\frac{2}{\circ}$ bind with oxidised haemoglobin resulting in lysis of $\underset{\Rightarrow}{\Rightarrow}$ red blood cells and intravascular haemolysis and $\frac{0}{3}$ anaemia. Renal damage follows the extensive $\vec{J}$ haemolysis. Many other body organs are at risk after sludging of red cell debris within the microcirculation, the hypoxic effect of anaemia and haemolysis, and $\mathbb{\varnothing}$ possibly a direct toxic effect of the arsine itself. Thus? hepatic, myocardial, neurological, and marrow damage have been reported and proved histologically. This case shows a further aspect of tissue necrosis hitherto not described, and that is skeletal muscle necrosis. A fourfold use in creatinine phosphokinase, $?$ with a normal $\mathrm{MB}$ fraction, and a twofold use in the $?$ aldolase concentrations were shown. The high concentrations of these enzymes together with severe my- $N$ algia suggest muscle necrosis and thus ${ }_{N}$ rhabdomyolysis with myoglobinuria may have contributed to the renal failure in this patient. This form $\mathrm{\omega}$ of renal damage could theoretically occur in all patients owing to the generalised tissue necrosis aftero exposure to arsine gas.

This case also clearly shows how marrow sup- $\stackrel{\mathscr{?}}{+}$ pression by arsine can give the impression that no or $\frac{T}{7}$ minimal haemolysis is taking place. The initial reticu- $\frac{O}{\mathbb{Q}}$ locyte counts suggested that severe continuous hae- 
molysis was not occurring. As a result the patient did not have immediate exchange transfusion. As his symptoms and renal function did not improve, however, we reviewed the position, and concluded that either arsine gas itself was still causing haemolysis, as evidenced by low haptoglobin and haemopexin levels, or that arsine complexes liberated by the lysis of red cells and themselves toxic to tissue were causing continuing damage. It is noteworthy that the patient showed dramatic improvement after the two belated exchange transfusions.

This case emphasises an important aspect of arsine poisoning. To arrest the processes involved in the tissue damage the arsine must be removed from the blood as soon as possible. Arsine is excreted in the urine. Therefore, if the patient is passing normal volumes of urine a forced diuresis should be implemented as soon as possible. In more severely affected cases where renal failure and anuria or oliguria are present other means of removing arsine must be used. As arsine haemoglobin complexes cross the peritoneal membrane, important in peritoneal dialysis, and cellophane membranes, used in extracorporeal haemodialysis, poorly, only small amounts of free arsine will be removed by these means. In acute renal failure only exchange transfusions will remove arsine and prevent continuing tissue damage. We recommend, as has been intimated previously, ${ }^{8}$ that early exchange transfusion is mandatory in renal failure due to arsine gas poisoning.
It is important to realise that other workers in the same workplace may be exposed to varying concentrations of arsine gas. If this form of poisoning is suspected screening programmes to detect subclinically affected individuals should be instituted. Laboratory evidence of haemolysis, including a urine test, to determine the presence of haemoglobinuria would be an adequate means of detecting exposed workers and forced diuresis would be sufficient to clear the arsine before it căn do much damage.

\section{References}

${ }^{1}$ Fowler BA, Weissberg JB. Arsine poisoning. New Engl J Med 1974;291:1171-4.

${ }^{2}$ Rathus E, Stinton RG, Putman JL. Arsine poisoning, country style. Med J Aust 1979;1:163-6.

${ }^{3}$ Parish GG, Glass R, Kimbrough R. Acute arsine poisoning in two workers cleaning a clogged drain. Arch Environ Health 1979;34:224-7.

${ }^{4}$ Kleinfeld MJ. Arsine poisoning. J Occup Med 1980;22:820-21.

${ }^{5}$ Williams PL. Suspected arsine poisoning during the restoration of a large cyclorama painting. Am Ind Hyg Assoc 1981;42:911-3.

${ }^{6}$ Levy H, Lewin HR, Ninin DT, et al. Asymptomatic arsine nephrotoxicity. A case report. S Afr Med J 1979;56:192-4.

${ }^{7}$ Muehrcke RC, Pirani CL. Arsine induced anuria. A correlative clinico-pathological study with electron microscopic observations. Ann Intern Med 1968;68:853-66.

${ }^{8}$ Teitelbaum DT, Kier LC. Arsine poisoning. A report of five cases in the petroleum industry and a discussion of the indications for exchange transfusions and haemodialysis. Arch Environ Health 1969;19:133-43. 